\title{
Illustrierte Presse für den republikanischen Bauern »Le Père Gérard. Gazette nationale des communes« (1878-1887)
}

\section{ENTSTEHUNG UND HISTORISCHER RAHMEN DER »GAZETTE NATIONALE«}

\begin{abstract}
Bei einer Betrachtung der Mediengesellschaft im Frankreich des 19. Jahrhunderts darf nicht aus dem Blick geraten, dass man es mit Veränderungsprozessen innerhalb einer mehrheitlich ländlichen Bevölkerung zu tun hat: 1876 lebten noch 67\% der Franzosen auf dem Land, und erst nach dem Zweiten Weltkrieg sollte dieser Anteil auf unter 50\% sinken ${ }^{1}$.
\end{abstract}

In den ländlichen Regionen ging die Veränderung der Lese- und Informationskultur seit Mitte des 19. Jahrhunderts mit einer zunehmenden Deproletarisierung und einer damit in Verbindung stehenden Alphabetisierung einher ${ }^{2}$. Während gleichzeitig zwischen 1850 und 1870 ein Großteil der auf Kolportageliteratur und »canards« spezialisierten Verlage - und mit ihnen die Kolporteure - verschwanden bzw. ihre Produktion umstellten ${ }^{3}$, wurden illustrierte, in Paris verlegte Zeitungen wie »Le Petit Journal« zunehmend auch auf dem Land gelesen und abonniert ${ }^{4}$. Seit den 1860er Jahren wurden in Paris im Zuge dieser Entwicklung auch illustrierte Wochenzeitungen herausgegeben, die sich speziell an die außerhalb der Hauptstadt lebende Bevölkerung richteten. Auch Elphège Boursin, Redakteur und geistiger Vater des »Père Gérard «5,

1 Jean-Pierre JESSENNE, Les Campagnes françaises entre mythe et histoire (XVIII $-\mathrm{XXI}^{\mathrm{e}}$ siècle), Paris 2006, S. 79.

$2 \mathrm{Zu}$ diesen Prozessen vgl. James R. LeHning, Peasant and French: Cultural Contact in Rural France During the 19th Century, New York u.a. 1995, sowie Peter MCPHEE, The Politics of Rural Life. Political Mobilization in the French Countryside 1846-1852, Oxford 1992, S. 276: »the cumulative effects of these slow and uneven changes - rural exodus and >dedifferentiation<, greater prosperity for most of those who remained, the erosion of local languages and cultures - were to be felt in the nature of political life in the countryside «.

3 Laurence FonTAINE, Histoire du colportage en Europe. XV ${ }^{\mathrm{e}}-\mathrm{XIX}^{\mathrm{e}}$ siècle, Paris 1993, S. 189, sowie Elisabeth PARINET, Une histoire de l'édition à l'époque contemporaine ( $\mathrm{XIX}^{\mathrm{e}}-\mathrm{XX}^{\mathrm{e}}$ siècle), Paris 2004, S. 123.

4 Vgl. Eugen WeBER, Peasants into Frenchmen. The Modernization of Rural France 1870-1914, London 1977, S. 466, sowie Anne Moulin, Les paysans dans la société française. De la Révolution à nos jours, Paris 1988, S. 151. Allg. zur Popularisierung der illustrierten Presse vgl. Jean-Pierre BACOT, La presse illustrée au XIX ${ }^{\mathrm{e}}$ siècle. Une histoire oubliée, Limoges 2005, S. 109-130.

5 Elphège Boursin wurde 1836 in Falaise geboren und starb 1891 in Paris. Als junger Journalist ging er rasch in Opposition zum Kaiserreich. Neben der Chefredaktion oder Mitarbeit an verschiedenen Zeitschriften und Zeitungen verfasste Elphège Boursin historische und ökonomische Werke, Wörterbücher, Theaterstücke und anderes mehr. Vgl. Michel PrEvost, JeanCharles Roman D’Amat (dir.), Dictionnaire de biographie française, Bd. 7, Paris 1956, S. 18. 
arbeitete von 1867 bis 1870 an einer solchen Zeitung mit, der »Gazette de Paris. Journal illustré des villes et des communes de France « ${ }^{6}$.

Mit seiner 1878 gegründeten und in Paris verlegten Wochenzeitschrift »Le Père Gérard. Gazette nationale des communes« wollte Boursin jedoch explizit die bäuerliche Landbevölkerung ansprechen ${ }^{7}$ und fand so für sein reges Interesse an der Modernisierung der ländlichen Regionen ein neues Ausdrucksmittel ${ }^{8}$.

Der Titel der Zeitschrift bezieht sich explizit auf den von Collot d'Herbois während der Französischen Revolution verfassten »Almanach du Père Gérard«. Collot d'Herbois hatte im September 1791 einen von der Société des amis de la Constitution ausgeschriebenen Wettbewerb für ein leicht verständliches Werk gewonnen, das breite Bevölkerungsschichten für die Verfassung gewinnen sollte. Um die Verbreitung zu erhöhen, wurden 1792 neben der französischen auch Ausgaben in bretonischer, provençalischer und flämischer Sprache gedruckt. Abgesehen von einigen unverzichtbaren kalendarischen Informationen war der »Père Gérard« kein Almanach im eigentlichen Sinne. Essenzieller Bestandteil der insgesamt acht Ausgaben waren die Gespräche über republikanische Institutionen und Tugenden, die Michel Gérard alias Père Gérard, Bauer und Abgeordneter der Nationalversammlung, nach deren Auflösung in seinem Dorf im Departement Ille-et-Vilaine mit den Bauern führt ${ }^{9}$. Die Wiederbelebung der Figur des Père Gérard unter der III. Republik schreibt sich bewusst in diese Tradition ein. Alle republikanischen Texte, die Boursin seit Gründung

6 Ab 1870 war Boursin Chefredakteur der in Besançon dreiwöchentlich erscheinenden Zeitung »L'Est. Journal démocratique«, bis diese nach Ausrufung des état de siège am 21. Juli 1871 eingestellt wurde. Nach dem Krieg arbeitete er für die kurzlebige Zeitung »Courrier de Paris et des départements. Journal des villes et des communes de France«, die im Februar 1872 in drei Ausgaben erschien.

7 Im Gegensatz zur Buch- und Almanachlektüre hatten Zeitschriften bei der Landbevölkerung noch keine große Beachtung gefunden. Vgl. Martyn LyONS, What Did the Peasants Read? Written and Printed Culture in Rural France, 1815-1914, in: European History Quarterly 27 (1997), S. 165-197.

8 Vgl. Elphège Boursin, Histoire de l'agriculture, du commerce et de l'industrie depuis le commencement de la monarchie jusqu'à nos jours précédée d'études historiques sur le commerce des peuples anciens, Paris 1867, S. 155: »La vapeur, l'électricité, les chemins de fer ont profité à l'agriculture comme des importantes découvertes ont profité à l'industrie, au commerce et à la civilisation. Les rapports sont devenus plus fréquents, les voyages plus faciles et moins coûteux, les communications plus rapides«.

$9 \mathrm{Zu}$ Collot d'Herbois' »Almanach du Père Gérard « vgl. Michel BIARD, L'Almanach du Père Gérard, un exemple de diffusion des idées jacobines, in: Annales historiques de la Révolution française 283 (1991), S. 19-29; Guy LemarCHAND, L'Almanach du Père Gérard de J.-M. Collot d'Herbois (1791), in: Annales historiques de la Révolution française 341 (http://ahrf.revues.org/ document2229.html). Zu den bretonischen Übersetzungen vgl.: L'Almanach du Père Gérard de J. M. Collot d'Herbois (1791). Le texte français et ses deux traductions en breton édités et annotés par Gwennole LE MENN. Préface et commentaires de Michel BIARD, Saint-Brieuc 2003 (Bibliothèque bretonne, 14). Darin auch: Michel BIARD, Le contexte historique de l'Almanach du Père Gérard, S. 11-25. Zur Figur des Erzählers vgl. Hans-Jürgen LÜSEBRINK, Du Messager Boiteux au Père Gérard: Les figures de narrateurs populaires dans les almanachs, XVIII - XIX ${ }^{\mathrm{e}}$ siècles (texte et iconographie), in: Jacques MigOZZI (Hg.), De l'écrit à l'écran. Littératures populaires: mutations génériques, mutations médiatiques, Limoges 2000, S. 53-57. Die Neuauflagen des »Almanach du Père Gérard« ab 1871 werden nicht berücksichtigt. 
der III. Republik verfasste, standen im Zeichen des »Père Gérard«: So erschien ab 1871 zunächst als direkte Wiederaufnahme der jährlich neu aufgelegte »Almanach du Père Gérard «, der bis 1892 existierte. 1872 wurde die Reihe der »Politique du Père Gérard« gegründet, deren erster Band, der »Catéchisme du bon Républicain« ${ }^{10}$, innerhalb von zwei Jahren fünfmal neu aufgelegt wurde.

Die Figur des Père Gérard entwickelte sich dabei von der eines republikanischen Tagelöhners allmählich zu der eines allwissenden Übervaters, der die Landbewohner besucht, ihnen Neuigkeiten überbringt und sie belehrt.

Der Gründungszeitpunkt der Zeitschrift im Herbst 1878 fiel in ein Schlüsselmoment der Dritten Republik: Auf der am 1. Mai 1878 in Paris eröffneten Weltausstellung wurde das Bild einer starken Republik präsentiert, die das Land nach Krieg und Commune zu neuem Glanz geführt hatte. Auch in den ersten Ausgaben der "Gazette nationale« wird regelmäßig über die Weltausstellung, Indiz für den wirtschaftlichen Aufschwung, berichtet und vor allem ihre Überlegenheit gegenüber der im Zeichen des II. Empire stehenden Ausstellung von 1867 betont $^{11}$.

Im selben Zeitraum verfestigte sich jedoch eine langwierige Agrarkrise, die auf teils verheerenden Schädlingsbefall und Missernten zurückging und so zum Anstieg des Imports landwirtschaftlicher Produkte führte ${ }^{12}$. Zwischen 1875 und 1896 sollten allein die Getreide- und Kartoffelpreise um über 30\% zurückgehen. Zwar waren die Zeiten verheerender Hungerkrisen vorbei, doch es kam zu einer langfristigen Stagnation der landwirtschaftlichen Einkommen, die in der Phase wirtschaftlicher Prosperität seit den 1850er Jahren zwanzig- bis siebzigprozentige Steigerungen verzeichnet hatten ${ }^{13}$. Seit 1871 hatte die ländliche Bevölkerung mehrheitlich republikanisch gewählt, so auch bei den Regionalwahlen im Januar 1878, die zu einer republikanischen »révolution des mairies « geführt hatte ${ }^{14}$. Dieser Rückhalt schien nun in Gefahr zu sein und den Befürwortern der Monarchie in die Hände zu spielen. Auf nationaler Ebene führte dies zu einer Stärkung der Agrarpolitik. Die Forderungen vor allem der Getreidebauern nach einer restriktiven Einfuhrpolitik mündeten mittelfristig - bis etwa 1882 - in der Schaffung der protektionistischen Agrarpolitik durch Léon Gambetta. Dies wurde gleichzeitig für den republikanischen Mythos insofern nutzbar gemacht, als eine direkte Verbindung zwischen Republik und Protektionismus wie auf der anderen Seite zwischen Kaiserreich und importorientiertem Freihandel geschaffen wurde ${ }^{15}$. Zudem wurde - aufgrund der Abspaltung des radikalen Flügels innerhalb des Parti républicain und des Verlusts urbaner Wählerschichten - der Prozess einer ideologischen

10 La politique du père Gérard. Catéchisme du bon Républicain par Elphège BouRSIN, rédacteur du Courrier de Paris. André Sagnier, éditeur, carrefour de l'Odéon, 7, à Paris. Se trouve chez tous les librairies dans toutes les gares de chemins de fer. 1872.

11 Vgl. u.a. Gazette nationale Nr. 4, 21.-28.10.1878.

12 Vgl. JESSENNE, Les Campagnes françaises entre mythe et histoire (wie Anm. 1), S. 196-198; MOULIN, Les paysans dans la société française (wie Anm. 4), S. 118f.

13 JESSENNE, Les Campagnes françaises entre mythe et histoire (wie Anm. 1), S. 184.

14 Maurice Agulhon, Apogée et crise de la civilisation paysanne, 1789-1914, Paris 1976 (Histoire de la France rurale, 3), S. 373f.

15 Soo-Yun Chun, Amis de l'agriculture (1870-1892) ou comment rallier les campagnes à la République?, in: Histoire et sociétés rurales 20 (2003), S. 147-172, hier S. 164, 170. 
$»$ Ruralisierung « der Republik beschleunigt ${ }^{16}$. Die sensible Phase vor der Umsetzung des Agrarprotektionismus, in der die Bauern sich von der Republik mit der Krise im Stich gelassen fühlten, bildet also den unmittelbaren zeitlichen Rahmen für die Gründung der »Gazette nationale «.

Günstig war zudem, dass die restriktive Pressepolitik des ordre moral infolge der republikanischen Wahlerfolge von 1878 wieder gelockert worden war. Fast zeitgleich aber war ein erstaunlicher Rückgang bei der Produktion und Verbreitung gedruckter republikanischer Propaganda im ländlichen Raum festzustellen. Dies galt vor allem für die seit 1871 auf diesem Gebiet äußerst aktive Société d'instruction républicaine, die ihre Tätigkeiten schon im Sommer 1877 weitgehend eingestellt hatte ${ }^{17}$.

Für die Verbindung von republikanischer Propaganda mit dem Typus der illustrierten Zeitschrift bestand also sowohl eine Marktlücke als auch Nachfrage. Nur somit ist zu erklären, dass die »Gazette nationale«, die mit einem Umfang von zwölf bis sechzehn Seiten zehn Centimes kostete - und damit erheblich teurer war als die etwa zeitgleich aufkommenden »quotidiens à un sou « ${ }^{18}$ - über einen Zeitraum von neun Jahren ihre Leser fand.

\section{DISKURSIVE UND VISUELLE STRATEGIEN DER REPUBLIKANISIERUNG IN DER »GAZETTE NATIONALE«}

Die zentrale rhetorische Strategie der Zeitschrift war die der direkten Ansprache, die sowohl auf der Text- als auch auf der Bildebene konsequent durchgeführt wurde. Dieses Prinzip kennzeichnet der Herausgeber schon in der ersten Nummer der »Gazette« vom 30. September 1878 als »La Politique du Père Gérard«: In einem fiktiven Dialog lässt sich Père Gérard von seinen amis villageois gleichsam entlarven, die ihn sofort als Verfasser der famosen republikanischen Broschüren wiedererkennen und ihm gute Aufnahme zusichern. Der Anschein des direkten Kontaktes wird auf der Bildebene weitergeführt. Die Gestaltung der ständigen Titelvignette, die Père Gérard im Kreise der um ihn versammelten Bauern zeigt, nimmt noch unmittelbar auf die Stiche Bezug, die in der Nachfolge von Collot d'Herbois' Almanachen entstanden ${ }^{19}$.

Die Illustrationen der »Gazette nationale« gehen aber über dieses Schema hinaus, indem sie zeigen, wie der neue Père Gérard die Bauern auf dem Feld besucht und sie dazu bringt, ihre Arbeit zu unterbrechen, wodurch der Eindruck einer unverzüglichen

16 Sudhir Hazareesingh, The Société d'instruction républicaine and the Propagation of Civic Republicanism in Provincial and Rural France, 1870-1877, in: Journal of Modern History 71 (1999), S. 271-307, hier S. 277. Allg. dazu auch LeHNING, Peasant and French (wie Anm. 2).

17 HazAREESINGH, The Société d'Instruction Républicaine (wie Anm. 16), S. 301f.

18 Vgl. Marc MARTin, La presse régionale: des affiches aux grands quotidiens, Paris 2002, S. $140 \mathrm{f}$.

$19 \mathrm{Im}$ Besitz der BnF, Est. befindet sich u.a.: Le Père Gérard expliquant la Constitution à ses concitoyens (Anonym, Paris 1792). 
Vermittlung von aktueller Information entsteht, vom Mythos der Leibhaftigkeit des Père Gérard ganz zu schweigen.

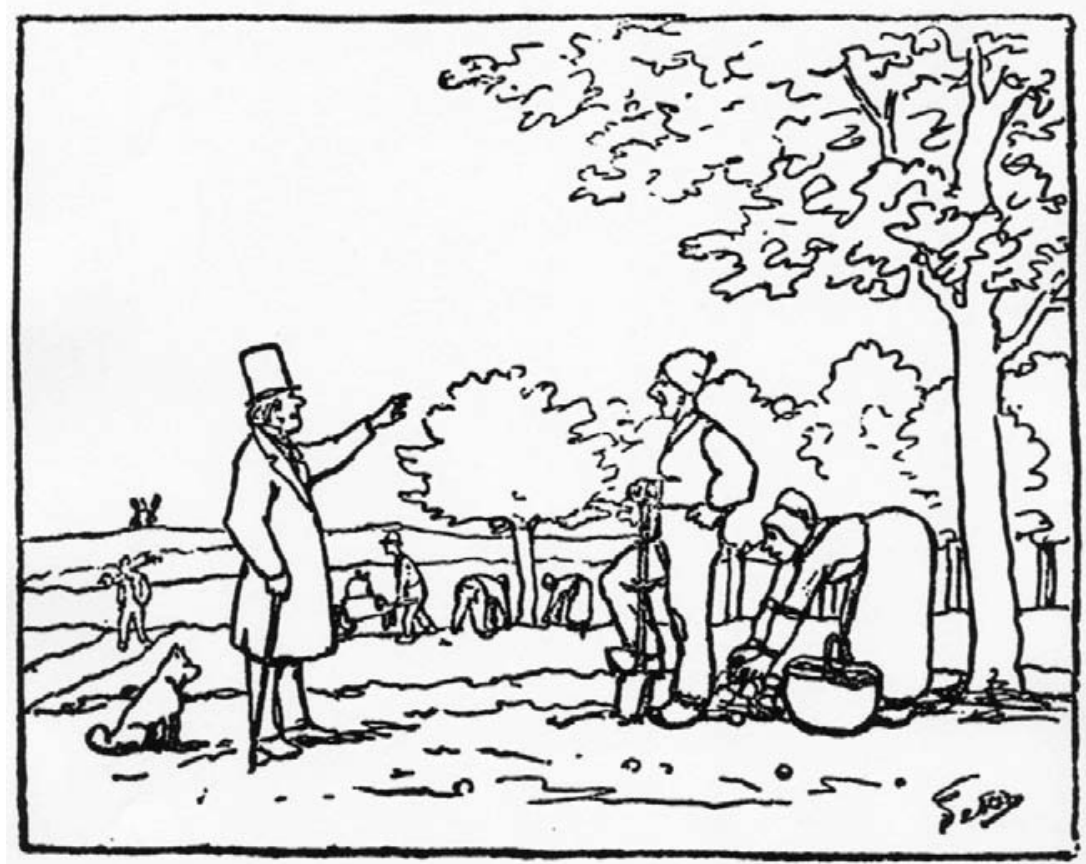

Abb. 1: Gazette nationale Nr. 5, 1878, Der Père Gérard besucht Bauern auf dem Feld.

Die so visualisierte Kommunikation auf Augenhöhe will sich zudem in eine oral geprägte ländliche Kultur einschreiben und von dieser profitieren: Die visuelle Thematisierung des Weitererzählens stellt eine unmittelbare Aufforderung zur Nachahmung dar.

Der direkte Kontakt wird in Gruppendiskussionen und fiktiven Korrespondenzen als den bevorzugten Formen der politischen Propaganda fortgesetzt. Die unmittelbare politische Belehrung findet in erster Linie durch den Briefwechsel Père Gérards mit einem gewissen Mathurin Heurtaud aus Ronfeugeray ${ }^{20}$ statt, laboureur und Gemeinderat, später auch Wahlmann für die Senatswahlen. Père Gérard legt seinem um Rat suchenden Freund Antworten in den Mund, die dem Leser somit als vorfabrizierte Argumente für die Republik zugänglich gemacht werden. In Mathurins Kontakten mit den ländlichen Wählern, die er in seinen Briefen an Père Gérard detailreich und weit-

20 Ronfeugeray ist in der »Lettre à mon Député« (1872) der Wohnort des Père Gérard, der als Tagelöhner in einem Brief seiner Sorge um die Zukunft der Republik Ausdruck verleiht. 
schweifig schildert, wiederholt der Lokalpolitiker oft wörtlich, was ihm Père Gérard im vorigen Brief geraten hat. Die Gesprächspartner, sofern republikanischer Gesinnung, sind ihrerseits natürlich selbst eifrige Leser der Schriften des Père Gérard, dessen Lektionen sie bei jeder sich bietenden Gelegenheit, oft unter Hinweis auf die Quelle, zitieren. Durch derartige Wiederholungen werden dem Leser die republikanischen Bekenntnisse regelrecht eingebläut. Ähnlichkeiten mit dem Elementarschulwesen sind unverkennbar.

Gleichzeitig berichtet Mathurin von den Intrigen eingeschworener Republikgegner, die sein Engagement hintertreiben oder seinen Platz einnehmen wollen. Die Briefe Mathurins an Père Gérard lesen sich somit als spannende Fortsetzungsgeschichte, die den Leser an die Zeitung binden soll.

Vor dem skizzierten politischen Hintergrund war es eine der grundlegenden Strategien der »Gazette nationale des communes«, die Republik von jeglicher Verantwortung für die wirtschaftlichen Schwierigkeiten freizusprechen und diese als Folge des durch das Kaiserreich verschuldeten Krieges darzustellen. Mehr als einmal gibt Père Gérard seinem Briefpartner Mathurin Heurtaud entsprechende Antworten für die Bonapartisten vor, die stets mit Bekenntnissen enden wie: »La République est à jamais implantée en France! «21.

»Republikanisierung« ist im »Père Gérard« also mit »Entnapoleonisierung« gleichzusetzen: Aus $» L ' E m p i r e$, c’est la paix « ${ }^{22}$ wird $» L a$ République, c'est la paix «23. Père Gérard will dabei den Bauern vor Augen führen, dass sie vor dem Plebiszit von 1852 von einer geschickt angelegten Medienkampagne indoktriniert wurden:

Des cahiers de chansons inondèrent les villages et les villes, et Napoléon y rimait toujours avec canon, et victoire avec gloire [...]. Nos braves habitants des campagnes, qui n'y voyaient pas malice, et qui à cette époque, il faut bien le dire, étaient beaucoup moins instruits des affaires politiques qu'aujourd'hui, [...] faisaient chorus avec les vieux grognards, et le nom de Napoléon devint de plus en plus populaire ${ }^{24}$.

Damit wird einerseits einer Affinität der Bauern zum napoleonischen Mythos, wie von Karl Marx vertreten, widersprochen, andererseits werden die Leser dazu angehalten, die Lektüre der »Gazette nationale« als Teil dieses Aufklärungsprozesses wahrzunehmen. Dadurch begibt sich die »Gazette nationale« auf eine Position moralischer Unangreifbarkeit, die sie gleichzeitig über den Bereich medialer Manipulation hinaushebt und verhindern soll, dass die neu erworbene Medienkompetenz kritisch auf die Lektüre der »Gazette nationale« angewendet wird.

Die auf den ersten Blick schlichte Bildsprache erweist sich bei näherem Hinsehen als eine damit eng verknüpfte, ausgefeilte und systematische Substitution von Symbolen, die auf die gesamte Lebenswirklichkeit der Zielgruppe ausgedehnt wird. Dies wird

21 Gazette nationale Nr. 4, 21.-28.10.1878.

22 Ausspruch Louis Napoléons vom 9. Dezember 1853: „Certaines personnes se disent, par esprit de défiance: >l'Empire, c'est la guerreく. Moi, je dis: l'Empire, c'est la paix «.

23 Titelseite der Gazette nationale, 5.9.1880.

24 Ibid., Nr. 10, 2.-9.12.1878, S. 110f. (Die Seiten wurden über mehrere Nummern durchlaufend gezählt.) 
durch den extensiven Gebrauch der Sonnensymbolik erreicht: Bilder wie »C'est la lumière qui commence à les éclairer (1878) übertragen die lebensspendende Funktion der Sonne explizit auf die Republik und heben zudem die traditionelle Aufklärungssymbolik hervor.

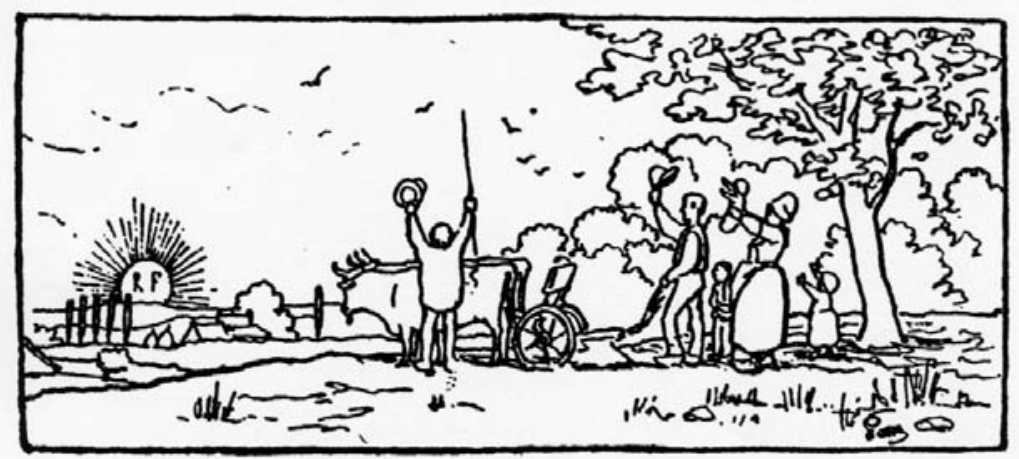

C’est la lumière qui commence a les éclairer.

Abb. 2: Gazette nationale, C'est la lumière qui commence à les éclairer (1878).

Die Lichtsymbolik, zeitgleich weit verbreitet in den zahlreichen Pariser Satiremagazinen ${ }^{25}$, wird hier insofern konkretisiert, als die dargestellte Situation fast alltäglich wirkt und sich daher stark von der oft sehr komplexen Symbolik der politischen Karikatur von Zeitschriften wie »Le Charivari« oder »Le Grelot« unterscheidet ${ }^{26}$. Die Republik ist in dieser Welt nicht nur immer und überall anwesend, sondern sie ist Lebensspenderin und erfüllt das Dasein mit Sinn.

So wird die personifizierte Republik in verschiedensten Illustrationen auch visuell in die Mitte der bäuerlichen Gesellschaft hineingeholt - sei es als Büste, sei es als leibhaftige Allegorie, wo sie gleichzeitig das Ideal der Einheit - im Sinne einer einzigen republikanischen Partei - versinnbildlicht. Mahnungen zur Einheit - »Union! Union! vous répète le Père Gérard « 27 - sind als Untertitel vielen Illustrationen, die Père Gérard im Kreise der Bauern zeigen, beigegeben.

Diese Appelle sind durchaus als immanente Drohgeste zu verstehen: Vor allem in der Wahlpropaganda unterstreicht das Blatt eindringlich, dass Bauer und Republik ohneeinander nicht existieren können ${ }^{28}$. Auf die zahlenmäßige Macht, welche die

25 Vgl. dazu: Daniela KNEISsL, Die Republik im Zwielicht: Zur Metaphorik von Licht und Finsternis in der französischen Bildpublizistik (1871-1914), München 2010 (Pariser Historische Studien, 88).

${ }^{26}$ Dasselbe gilt für die stilistische Einfachheit der Zeichnungen von Léonce Petit, die sich stark von seinen anderen Arbeiten unterscheiden.

27 Z.B. Gazette nationale, 20.7.1885.

28 Vor den Wahlen wurden die Namen der zu wählenden republikanischen Kandidaten stets in ausführlichen chroniques électorales aufgeführt. Darüber hinaus bot die Redaktion in Wahlpe- 
angesprochene Wählerschicht repräsentierte, wird niemals explizit aufmerksam gemacht, stattdessen wird dazu ermahnt, wachsam zu sein, um die Errungenschaften der Französischen Revolution, nämlich Bürger- und Besitzrechte für die Bauern, nicht wieder zu verlieren. Das Rad der Geschichte wird also gleichsam zurückgedreht. Auch die unsichere Anfangsphase, die die Republik in den 1870er Jahren durchlebt hatte, wird künstlich verlängert und dramatisiert.

Um die Bindung der Bauern an die Republik zu stärken, betont die »Gazette nationale« gleichzeitig die Vereinbarkeit von Republikanismus und ländlicher Tradition: Gerade in Bezug auf die Kirche geht die Zeitschrift behutsam vor und vermeidet pauschalen Antiklerikalismus. Ohne explizit vom Kirchgang abhalten zu wollen, wird vor allem an die Toleranz gegenüber nicht Praktizierenden appelliert. Zudem unterscheidet Père Gérard in seinen Gesprächen zwischen Priestern, die sich der Seelsorge widmen und in politischen Dingen zurückhalten sowie dem Zerrbild des republikfeindlichen Jesuiten. Während der erste sich in die republikanische Gemeinschaft einfügt, flieht der andere vor den Strahlen der republikanischen Sonne. Der republiktreue Priester jedoch entspricht wesentlich mehr dem realen Erscheinungsbild eines Dorfpfarrers als der lichtscheue Jesuit - für die »Gazette nationale« ein typischer Kompromiss.

Abb. 3: Gazette nationale, 23.7.1882, La grande fête nationale du 14 juillet.

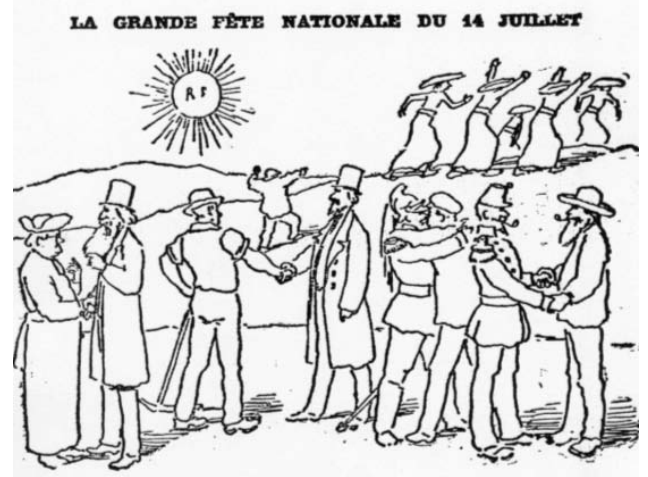

Ähnlich zweispurig wird bei der Scheidungsfrage verfahren. Einerseits hebt Père Gérard im Gespräch mit einer um die Moral fürchtenden Bäuerin hervor, dass die Scheidung unglücklich verheirateten und misshandelten Frauen zugutekäme ${ }^{29}$. Auf der wahrnehmungsintensiveren, visuellen Schiene triumphiert jedoch letztlich das Ideal der Großfamilie, die sich auf der Titelillustration vom 14. September 1882 dem Leser unter dem Motto »Rétablissement du divorce« präsentiert: »Divorcera qui voudra! Ma bonne Françoise et moi resterons ensemble au milieu de nos cinq enfants.«

rioden besonders preisgünstige zweimonatige Propagandaabonnements an, die interessierte Wahlkämpfer direkt und portofrei an die gewünschten Adressaten schicken lassen konnten.

${ }^{29}$ Gazette nationale, Nr. 38, 16.-23. Juni 1879. 
Mit derartigen Strategien werden die moralische Überlegenheit der Landbevölkerung und die Beständigkeit ihrer Werte eindeutig hervorgehoben. Stets geht es also darum, das Zielpublikum in der Sicherheit des Vertrauten zu wiegen und zu suggerieren, dass die Werte der bäuerlichen Gesellschaft zu den unverzichtbaren Grundlagen der Republik gehören.

Dies kommt exemplarisch in einer kleinen Vignette zum Ausdruck, die zu den am häufigsten gebrauchten Bildern in der Zeitschrift zählt: Die unscheinbare Zeichnung zeigt eine Glocke mit der Aufschrift »RF« - »République Française«, was vom Bewusstsein für die immense Bedeutung der Glocke für die Strukturierung des dörflichen Lebens zeugt ${ }^{30}$. Mehr als einmal beendet das Mittagsläuten die Gespräche des Père Gérard. Die Umwidmung der Glocke und ihres Geläutes zur "Stimme der Republik« beschreibt in eingängiger Art und Weise den festen Willen, die bäuerliche Gesellschaft von ihren Grundlagen her zu republikanisieren, zu kontrollieren und zu disziplinieren.

\section{ZUSAMMENFASSUNG}

Die »Gazette nationale« verschrieb sich ganz und gar der Aufgabe, die Republikanisierung der Bauern in einer Krisenzeit der Landwirtschaft voranzutreiben.

Zwar sind die Gespräche und Briefwechsel Père Gérards als unmittelbare Reflexe der politischen Aktualität angelegt, doch die Vermittlung von politischen Fakten hatte in der »Gazette nationale« wenig Raum.

Die Schlichtheit des politischen Weltbildes, das sowohl aus den Texten wie aus den Illustrationen spricht, zielte darauf ab, den Leser nicht zu verwirren oder zu überfordern. Die Zensur spielte hierbei kaum eine Rolle, denn der Tenor der Zeitschrift blieb auch nach dem Erlass des Pressegesetzes von 1881 unverändert.

Die »Gazette« betrieb also eine strikte Informationskontrolle, die vor allem die verwirrende Vielfalt der politischen Wirklichkeit, in erster Linie natürlich in Bezug auf den in sich zerstrittenen Parti républicain, von ihren Lesern fernhalten wollte.

Die protektionistische Agrarpolitik, deren Entstehung und Frühphase die »Gazette nationale« während ihrer Erscheinungszeit begleitete, bildet gleichsam das mediale Prinzip der Zeitschrift. Dabei wendet die »Gazette« sich an einen naiven ländlichen Leser, der nach Erkenntnissen der Leseforschung zu Beginn der 1880er Jahre gerade im Verschwinden begriffen war: der unerfahrene Leser nämlich, der seine seltene Lektüre als übergeordnete Autorität und somit als absolute Wahrheit begreift ${ }^{31}$. Diese Vorstellung findet ihre Entsprechung im Bild der Öffentlichkeit, an die sich die »Gazette nationale« wendet: Ein der Republik durchaus gewogener, aber leicht beschränkter Landbewohner, für den Information in spezifischer Weise aufbereitet werden muss.

30 Vgl. Alain CoRBIN, Die Sprache der Glocken. Ländliche Gefühlskultur und symbolische Ordnung im Frankreich des 19. Jahrhunderts, Frankfurt a.M. 1994. (ID., Les cloches de la terre. Paysage sonore et culture sensible dans les campagnes françaises au XIX ${ }^{\mathrm{e}}$ siècle, Paris 1994.)

31 Vgl. LyONS, What Did the Peasants Read? (wie Anm. 7), S. 168: „From the 1880s onwards, a decisive transformation occurred. The peasant was no longer a stranger to the printed and the written word, and at the same time, he or she was no longer a victim of its all-embracing power«. 
Gleichzeitig bestand eine zentrale Strategie der Zeitschrift darin, vorgeblich originäre Meinungsäußerungen aus der Mitte der bäuerlichen Gesellschaft zu präsentieren und publik zu machen.

Ein wichtiges Ziel bestand darin, der bäuerlichen Bevölkerung ein nationalisiertes Bild ihrer selbst überzustülpen, in dem regionale Unterschiede keine Rolle mehr spielen. Zudem konnte sich das Publikum als Teil einer durchweg ländlich geprägten Republik erleben, die bäuerliche Werte hochhält. Den fortschreitenden Veränderungen der ländlichen Strukturen versuchte die »Gazette nationale« also mit der Erschaffung eines Schutzraumes zu begegnen, dessen Bewahrung nichtsdestoweniger an die Verantwortung der Leser überstellt und von dem Maß ihrer republikanischen Gesinnung abhängig gemacht wird.

Als leicht lesbares, illustriertes Wochenmagazin, das sein Publikum direkt ansprechen wollte, konnte sich die »Gazette nationale« so über eine beachtliche Zeitspanne behaupten, bis sie im Jahre 1887 eingestellt wurde ${ }^{32}$. Angesichts der sich rapide entwickelnden und emanzipierenden Provinzpresse, die gegenüber den aus Paris kommenden Zeitungen einen erheblichen Aktualitätsvorsprung besaß33, konnte die Mischung aus anspruchsloser Unterhaltung und republikanischer Propaganda der »Gazette nationale« letztlich wohl nicht bestehen. Aufstieg und Verschwinden der »Gazette nationale « spiegeln somit in einzigartiger Weise die Entwicklung der Mediengesellschaft im bäuerlichen Milieu vor dem politischen Hintergrund der Dritten Republik.

32 Elphège Boursin starb vermutlich bereits 1886 .

33 MARTIN, La presse régionale (wie Anm. 18), S. 143. Boursin stellte sich dieser Konkurrenz bereits in den 1880 in loser Folge erscheinenden Pamphleten »Le Père Gérard à ses amis de Quimperlé«, in denen er die Artikel des in Quimperlé verlegten »Publicateur du Finistère« angriff. 